\title{
The CKM Lattice Working Group initiative*
}

\author{
Jonathan Flynn ${ }^{\mathrm{a}}$, Laurent Lellouch ${ }^{\mathrm{b}}$ and Guido Martinellic for the CKM-LWG

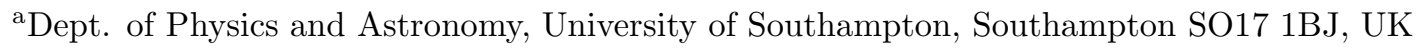

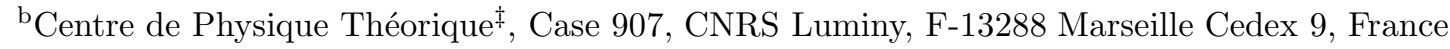 \\ ${ }^{\text {c} D i p . ~ d i ~ F i s i c a, ~ U n i v . ~ d i ~ R o m a ~ " L a ~ S a p i e n z a ", ~ P . l e ~ A . ~ M o r o ~ 2, ~ I-00185 ~ R o m e, ~ I t a l y ~}$
}

We present the CKM-LWG, a collection of working groups whose aim is to compile world averages of lattice results relevant for high-energy phenomenology.

\section{Short history}

In February 2002, the first meeting of the Workshop on the CKM Unitarity Triangle was held at CERN. The aim of the workshop is to review the state of the art in CKMology and propose avenues for new physics searches. Because lattice QCD has an important rôle to play in this program, the meeting was viewed by the authors as an excellent opportunity to bring together lattice theorists to begin setting up a $\mathrm{L}$ (attice) $\mathrm{D}$ (ata) G(roup) whose goal would be to compile averages for quantities calculated on the lattice which are relevant for high-energy phenomenology. Representatives from major lattice collaborations from around the world were thus invited to attend a first assembly on February 13 at CERN.

The response was certainly positive. The meeting was well attended [1] and most colleagues present shared the belief that it would be very helpful to join forces to combine results. Many also expressed the wish to contribute and to see something come out of this initiative.

A number of objections were raised, however. The first was that the project was too ambitious. Most lattice phenomenology results are still quenched or partially quenched and are, in that sense, still preliminary. Too close a parallel with the PDG, who compile experimental results, should thus be avoided. Another concern was manpower, especially if a large number of quanti-

\footnotetext{
*Plenary talk by L. Lellouch at Lattice 2002, Cambridge MA, USA. Work supported in part by EU HPP contract HPRN-CT-2000-00145.

$\ddagger$ Unité Propre de Recherche 7061 .
}

ties are to be reviewed all at once: an enterprise such as the LDG should not come at the expense of progress in our field. It was also noted that we, as a community, should build up some experience with this sort of compilation effort before embarking on a full-fledged PDG type enterprise.

After some discussion, it was concluded that the project should focus on a more manageable task and prove feasibility. It was agreed that three test working groups should be set up to review a number of well studied quantities:

- quark masses,

- the kaon $B$-parameter, $B_{K}$,

- matrix elements relevant for neutral $B$ meson mixing.

It was further agreed that only results published in refereed journals should be taken into account; that the lattice community as a whole should be informed about the project and given a chance to react and that wider participation should be solicited (this was the subject of an email sent to the mailing list of this conference last spring and is the purpose of the present talk and of the follow-on meeting); that the results of the analyses and the process which led to them should be presented at the first Lattice Symposium following their release. For the time being, the working groups are known collectively as the CKM Lattice Working Group (CKM-LWG).

\section{Why do we need a CKM-LWG?}

Lattice results already play an important rôle in a number of aspects of the current experi- 


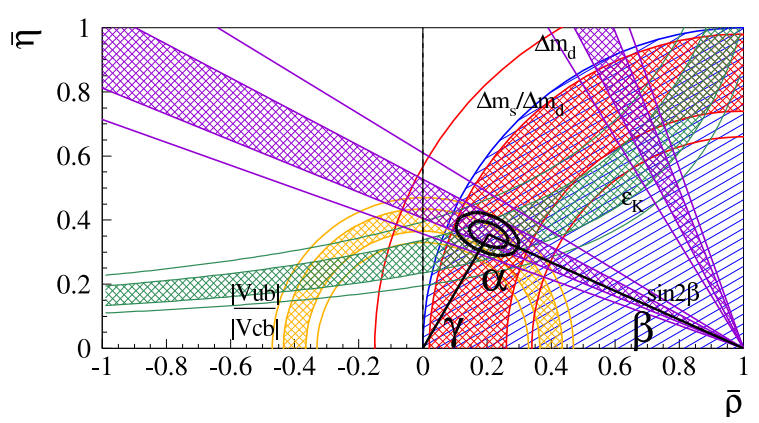

Figure 1. Constraints on the unitarity triangle (summer 2002) 国. The non-perturbative matrix elements required to constrain the sides and summit were taken from lattice calculations.

mental endeavor in high-energy physics. For instance, the discovery of new physics from analyses of the CKM unitarity triangle has come to rely more and more on weak matrix elements obtained through lattice calculations (please see Fig. 1). We thus have a responsibility as a community to provide reliable estimates of the relevant quantities and especially of their uncertainties: an underestimate could raise false hopes of new physics and discredit our field; an overestimate could mean that an unique opportunity to reveal new physics is lost.

Another important aspect is that for many quantities of phenomenological relevance, there exist a large number of calculations using different actions, different approaches to renormalization, etc. Furthermore, lattice theorists are curious to try out new approaches so that more recent does not necessarily imply more reliable or accurate. The situation can thus be very confusing for non-experts, as it is even for experts! It would therefore be very useful to have a body of theoreticians well versed in the different possible lattice approaches, which could review the situation and provide a succinct and reliable summary of the state of the art. We also owe it to ourselves to make the best possible use of the results and experience that we have accumulated.

\section{Isn't that the rôle of lattice rappor- teurs?}

Plenary speakers at lattice conferences are mainly expected to summarize and comment on the developments of the last year-many of which are still preliminary-and to give their views on future directions. Moreover, lattice plenaries can be rather technical and phenomenological information is often scattered over different reviews. This is to be contrasted with the focus of the CKM-LWG which is on providing the wider particle physics community with summary numbers for quantities of phenomenological interest which are based solely on results published in refereed journals (i.e. old news by lattice conference standards) and which are easily accessible.

Unlike plenary speakers at lattice symposia, the lattice rapporteur at large particle physics conferences is usually asked to put together a one hour or less summary of the situation in lattice field theory for subjects ranging from chiral fermions, through finite temperature phase transitions, to quark masses. In such a context, choices have to be made and it is clearly very difficult to provide a summary of the current situation for the whole range of phenomenologically relevant quantities. In contrast, each of the CKM Lattice Working Groups focuses on specific quantities and is composed of the physicists who have calculated and studied them. The working groups therefore have access to expertise in the different possible approaches as well as to details of the analyses, intermediate results, directions investigated but not retained etc. which can all be very helpful for optimally combining results from different calculations.

To conclude on this aspect, lattice reviews and the CKM-LWG are complementary: the CKMLWG should make use of the expertise put into lattice reviews and lattice reviews could use the work of the CKM-LWG as a standard against which new developments may be compared. A possible scenario would be to have the CKMLWG present its results in winter/spring so as to best carry out this complementarity; so as to allow time for the results presented at the lattice conference to make it to press; so as to provide 
updates in time for the phenomenological analyses presented in summer (spring?) conferences.

\section{Current status of the CKM-LWG}

The idea is to have up-to-date web pages which summarize the status of the CKM-LWG's work and which serve as an archive for past results [1. For the moment they contain the agenda of the CERN meeting, the CKM-LWG proposal and lists of the current membership of the three working groups. They also contain a set of suggested guidelines, which should give a flavor of the work required. The working groups should:

- compile all published results for a given quantity, specifying details of the calculation and systematic errors considered;

- determine a set of systematic errors ascribed to each quantity and each lattice approach and use the literature to estimate their size;

- on the basis of this information, exclude results for which these systematics are not under control and for the others, assign systematic errors which take into account the analysis choices made by authors;

- devise a means for combining the various results for a given quantity, distinguishing correlated and uncorrelated systematics. Results using similar approaches could first be combined and the resulting numbers further compiled into a global average;

- consult one another on common issues.

In addition, collaborations are strongly encouraged to make available any data which may help in the work of the different groups.

These guidelines are certainly not meant to be restrictive and each group should organize itself and its work as it sees fit. The experiences of the groups can then be compared and the best of each retained. This will hopefully lead to a set of generally accepted criteria for computing global averages, keeping in mind that different subjects sometimes require different approaches.

Some very preliminary work has begun. Two of the working groups, for instance, have set up a web page and some bibliographies have been collected. However, time was too short and schedules too full to produce even preliminary analyses for this conference.

\section{What else could the CKM-LWG do for our community?}

When presented with the idea, Karl Jansen made the pertinent remark that another rôle of the PDG is to provide concise and accessible reviews on subjects which are important for the every day work of particle physicists. Following this line of thought one can imagine collecting, say on a web page, information that would be useful to our community (including students in our field) as well as to others interested in understanding what we do, e.g.

- descriptions of the various algorithms used, including those for inversions, evaluating the overlap Dirac operator, etc.,

- estimates of the performances of these algorithms,

- a description of the basic fitting and analysis techniques,

- a summary of the different fermion and gauge actions used,

- plaquette values for different actions,

- reviews on different systematics,

- a review of the possible approaches to renormalization,

- a collection of perturbative expressions for matching factors for different actions and quantities,

- a collection of non-perturbative (NP) matching factors and NP lagrangian parameters for different actions and quantities.

The possibilities are numerous and having a unique repository for this kind of information could be very helpful.

One could also imagine making available gauge configurations, propagators, codes, etc. This, however, is a different business and a very interesting proposal along these lines [3], which advocates the creation of an "International Lattice Data Grid" (ILDG), was presented at this conference 四. 


\section{Conclusion}

A number of us believe that an initiative such as the CKM-LWG would be very useful for highenergy physics as well as for the lattice community. We are currently running a feasibility experiment with three working groups on:

- quark masses,

- $B_{K}$,

- $B-\bar{B}$ mixing,

and plan to produce a first CKM-LWG summary by the Lattice 2003 conference in Tsukuba at the latest. At that point, the experiences of each group will be compared, and decisions on where to go from there will be made.

The CKM-LWG, and perhaps later the LDG, can also serve our community by putting together information which is useful to our everyday work (reviews on algorithms, etc.). Work on this aspect could start now, though it should not divert (too many) resources from the phenomenological missions of the CKM-LWG.

In sum, possibilities are numerous and it is up to us to decide what we might want an LDG to be. Anyone interested in joining can contact the authors or any other member of the CKM-LWG.

\section{Note added}

In the evening following this talk, an open meeting was held to answer more detailed questions about the proposal and to discuss the rôle of the CKM-LWG. Points raised during this meeting which were not covered above are now briefly reviewed.

An important issue that was raised concerned the procedure by which the groups will reach a consensus on the different quantities studied, given the sometimes large systematic uncertainties and the prejudices that members may have regarding different possible approaches. It was suggested that each group should set up the rules by which decisions will be made before any final discussion about the summary results begins. The importance of having the process that leads to any conclusion public and open to scrutiny, to ensure that the people involved are accountable, was re-iterated. It was also emphasized that results should be archived to further promote accountability.

Another concern, already expressed during the morning session, was that citations for results would go to the CKM-LWG instead of to the original papers on which these results are based. To attenuate this problem, it was suggested that the CKM-LWG could provide on the web site, next to the various results presented, ready-made $\mathrm{L}_{\mathrm{E}} \mathrm{X}$ bibliographic entries of the form: "CKM-LWG 200x, A. Aardvark et al. (http://www.cpt.univ-mrs.fr/ldg/), obtained using the results from ...", with a complete list of the works used.

Finally, the idea of having three coordinators per group was upheld. However, since the members of the groups were not all present at the meeting, it was decided that one person from each group would be responsible for making sure that the coordinators get appointed: Vittorio Lubicz for the group on quark masses, Anastassios Vladikas for the group on $B_{K}$ and Hartmut Wittig for the one on $B$-meson mixing. It was further suggested that every member in a group should review all of the literature pertaining to the quantity under study.

L.L. thanks J. Christensen for sharing the notes which he took during the evening discussion at M.I.T. and L. Giusti for discussions about the project.

\section{REFERENCES}

1. http://www.cpt.univ-mrs.fr/ldg/

2. M. Ciuchini et al., paper ABS657 submitted to ICHEP 2002, Amsterdam, 2431 July 2002 (plot is courtesy of F. Parodi). Comparable analyses can be found at http://ckmfitter.in2p3.fr/, A. Höcker, H. Lacker, S. Laplace and F. Le Diberder, Eur. Phys. J. C 21 (2001) 225.

3. Richard Kenway, private communication.

4. C.T.H. Davies et al., this volume. 